\title{
ON FRACTIONAL DERIVATIVES OF UNIVALENT FUNCTIONS ${ }^{1}$
}

H. P. ATKINS

It has been shown by F. Marty [6] ${ }^{2}$ that if $f(z)$ is analytic and univalent in the unit circle, $f(0)=0$, and $f^{\prime}(0)=1$, and if the Bieberbach conjecture [2] that $\left|f^{(n)}(0)\right| \leqq n \cdot n$ ! is assumed when $n>3$, then

$$
\left|f^{(n)}(z)\right| \leqq n !(n+r)(1-r)^{-n-2}, \quad n=0,1,2,3, \cdots,
$$

where $|z|=r$, and that equality is attained for real positive $z$ by the function $f(z)=z(1-z)^{-2}$. For $n=0$ and $n=1$ the inequality reduces to the well known relations obtained by Pick in evaluating the constant in the Verzerrungssatz of Koebe (see [2]).

The purpose of this paper is to generalize this relation to include fractional derivatives and integrals. The bound obtained will be expressed in terms of the ratio of the incomplete to the complete beta function, defined for $p$ and $q$ real and one or the other positive by the equations

$$
\begin{aligned}
& I_{r}(p, q)=\frac{1}{B(p, q)} \int_{0}^{r} x^{p-1}(1-x)^{q-1} d x, \quad p>0, \\
& I_{r}(p, q)=1-\frac{1}{B(p, q)} \int_{r}^{1} x^{p-1}(1-x)^{q-1} d x, \quad q>0,
\end{aligned}
$$

which are equivalent if both $p$ and $q$ are positive. Two separate definitions of the fractional derivative will be found useful; these may be shown equivalent for the values for which both are defined. For $a<0$ the Abel-Riemann definition $[1,9]$ is more easily applied:

$$
\begin{aligned}
& { }_{0} D_{z}^{a} f(z)=\frac{1}{\Gamma(-a)} \int_{0}^{z} f(w)(z-w)^{-a-1} d w, \quad a<0, \\
& { }_{0} D_{z}^{a} f(z)=\frac{d^{m}}{d z^{m}}{ }_{0} D_{z}^{a-m} f(z), \quad \quad m-1 \leqq a<m,
\end{aligned}
$$

where $m$ is a positive integer. For $a \geqq 0$ the Laurent definition [4] is more satisfactory:

Presented to the Society, August 23, 1946; received by the editors April 17, 1946.

1 The material of this paper forms part of a thesis, prepared under Professor W. Seidel, to be presented to the Graduate School of The University of Rochester for the degree Doctor of Philosophy.

2 Numbers in brackets refer to the bibliography. 


$$
{ }_{0} D_{z}^{a} f(z)=\frac{\Gamma(a+1)}{2 \pi i} \int_{P} f(w)(w-z)^{-a-1} d w, \quad a \geqq 0,
$$

where $P$ is a path from 0 once around $z$ in the positive sense and returning to 0 . To avoid ambiguity in the Laurent definition we shall use that determination of the multiple valued quantity $(w-z)^{-a-1}$ obtained by letting

$$
(w-z)^{-a-1}=r^{-a-1} e^{-(t-\pi)(a+1) i},
$$

where $-\pi<t \leqq \pi$, at the start of the path $P$. The corresponding ambiguity in the Abel-Riemann definition will cause no difficulty. It should be noted that the fractional derivative may be undefined for $z=0$.

THEOREM. If $f(z)$ is analytic and univalent in the unit circle with $f(0)=0$ and $f^{\prime}(0)=1$, and if $a$ is any real number and $n+1$ the smallest non-negative integer greater than $a$, then (without restriction if $a \leqq 3, b u t$ assuming the Bieberbach conjecture if $a>3)^{3}$

$$
\begin{aligned}
& \left|{ }_{0} D_{z}^{a} f(z)-\sum_{j=1}^{n}{ }_{0} D_{z}^{a-j} f^{(j)}(0)\right| \\
& \quad \leqq(1-r)^{-a-2} \Gamma(a+1)\left\{a I_{r}(n+1-a, a+2)+r I_{r}(n-a, a+2)\right\}
\end{aligned}
$$

for $z \neq 0$. Equality is attained for real positive $z$ by the function $f(z)$ $=z(1-z)^{-2}$.

(The summation from 1 to $n$ is to be interpreted as 0 if $n<1$, and ${ }_{0} D_{z}^{a-j} f^{(j)}(0)$ means the derivative of order $a-j$ of the constant $f^{(j)}(0)$.)

PROof. If $a<0$, the desired relation follows from the Abel-Riemann definition, for making the substitutions

$$
w=\frac{x-1+r}{x} e^{i t}, \quad z=r e^{i t}
$$

and noting that, from equation (1) with $n=0$,

$$
\left|f\left(\frac{x-1+r}{x} e^{i t}\right)\right| \leqq x^{2}(1-r)^{-2}-x(1-r)^{-1},
$$

we have

8 Using the Littlewood result [5] that $\left|f^{(n)}(0)\right|<e n \cdot n$ !, the theorem may be changed so as to be valid without assuming the Bieberbach conjecture by inserting a factor $e$ on the right side of the relation, making the relation a definite inequality, and deleting the last sentence. 


$$
\begin{aligned}
&\left|{ }_{0} D_{z}^{a} f(z)\right| \leqq \frac{1}{\Gamma(-a)} \int_{1-r}^{1}(1-r)^{-a-2} x^{a}(1-x)^{-a-1}(x-1+r) d x \\
&=(1-r)^{-a-2} \Gamma(a+1)\left\{(a+1) I_{r}(-a, a+2)-(1-r) I_{r}(-a, a+1)\right\} .
\end{aligned}
$$

Using the reduction formula [8]

$$
I_{r}(p, q)=r I_{r}(p-1, q)+(1-r) I_{r}(p, q-1),
$$

which may easily be verified for all cases, we obtain the desired result for $n=-1$,

$$
\begin{aligned}
& \left|{ }_{0} D_{z}^{a} f(z)\right| \\
& \quad \leqq(1-r)^{-a-2} \Gamma(a+1)\left\{a I_{r}(-a, a+2)+r I_{r}(-1-a, a+2)\right\} .
\end{aligned}
$$

If $a \geqq 0$ we use the Laurent definition of the fractional derivative. Let $b=a-n$, where $n=[a]$, and temporarily restrict $b$ to be different from 0 . Let $g(z)=f^{(n)}(z)-f^{(n)}(0)$, so that $g(z)$ is analytic and $g(0)=0$.

Select $c$ between 0 and $r$, and take the path of integration $P$ to consist of a straight line from 0 to $(r-c+c r) e^{i t}$, a circle with $z$ as center traversed once in the positive sense, and a straight line back to 0 .

On the circular portion of this path, putting $w=z+c(1-r) e^{i \theta}$ and integrating by parts with $e^{-i b \theta} d \theta$ as the differential,

$$
\begin{aligned}
& \frac{\Gamma(b+1)}{2 \pi i} \int g(w)(w-z)^{-b-1} d w \\
& =\frac{1}{\Gamma(1-b)}(1-r)^{-b} c^{-b} e^{-b i t} g\left([r-c+c r] e^{i t}\right)+O\left(c^{1-b}\right),
\end{aligned}
$$

where $c^{b-1} O\left(c^{1-b}\right)$ is bounded, for fixed $z$, as $c$ tends to 0 .

On the two straight portions of the path $P$, taken together,

$$
\begin{aligned}
& \frac{\Gamma(b+1)}{2 \pi i} \int g(w)(w-z)^{-b-1} d w \\
& =\frac{\Gamma(b+1)}{2 \pi i} \int_{0}^{r-c+c r} g\left(|w| e^{i t}\right)(r-|w|)^{-b-1} e^{-(b+1)(t-x) i} e^{i t} d|w| \\
& \quad+\frac{\Gamma(b+1)}{2 \pi i} \int_{r-c+c r}^{0} g\left(|w| e^{i t}\right)(r-|w|)^{-b-1} e^{-(b+1)(t+\pi) i} e^{i t} d|w| \\
& =\frac{1}{\Gamma(-b)} e^{-b i t} \int_{0}^{r-c+c r} g\left(|w| e^{i t}\right)(r-|w|)^{-b-1} d|w| .
\end{aligned}
$$

The substitution $|w|=(x-1+r) / x$ and integration by parts with $(1-x)^{-b-1} d x$ as the differential give 


$$
\begin{aligned}
& -\frac{1}{\Gamma(1-b)} e^{-b i t}(1-r)^{-b}(1+c) c^{-b} g\left([r-c+c r] e^{i t}\right) \\
& +\frac{1}{\Gamma(1-b)} e^{-b i t}(1-r)^{-b}(b-1) \int_{1-r}^{1 /(1+c)} x^{b-2}(1-x)^{-b} g\left(\frac{x-1+r}{x} e^{i t}\right) d x \\
& +\frac{1}{\Gamma(1-b)} e^{(1-b) i t}(1-r)^{1-b} \int_{1-r}^{1 /(1+c)} x^{b-3}(1-x)^{-b} g^{\prime}\left(\frac{x-1+r}{x} e^{i t}\right) d x
\end{aligned}
$$

Combining this with the value obtained on the curved portion of $P$, and allowing $c$ to approach 0 , we have

${ }_{0} D_{z}^{b} g(z)$

$$
\begin{aligned}
= & \frac{1}{\Gamma(1-b)} e^{-b i t}(1-r)^{-b}(b-1) \int_{1-r}^{1} x^{b-2}(1-x)^{-b} g\left(\frac{x-1+r}{x} e^{i t}\right) d x \\
& +\frac{1}{\Gamma(1-b)} e^{(1-b) i t}(1-r)^{1-b} \int_{1-r}^{1} x^{b-3}(1-x)^{-b} g^{\prime}\left(\frac{x-1+r}{x} e^{i t}\right) d x .
\end{aligned}
$$

Integrating the first integral by parts, with $x^{b-2}(1-x)^{-b} d x$ as the differential, gives finally

$$
\begin{aligned}
& { }_{0} D_{z}^{b} g(z) \\
& \quad=\frac{1}{\Gamma(1-b)} e^{(1-b) i t}(1-r)^{1-b} \int_{1-r}^{1} x^{b-2}(1-x)^{-b} g^{\prime}\left(\frac{x-1+r}{x} e^{i t}\right) d x .
\end{aligned}
$$

This formula may be verified directly for $b=0$ so the restriction on $b$ may be removed. If we note that, by univalence of $f(z)$, formula (1) gives (see also [7])

$$
\begin{aligned}
\left|g^{\prime}\left(\frac{x-1+r}{x} e^{i t}\right)\right| & =\left|f^{(n+1)}\left(\frac{x-1+r}{x} e^{i t}\right)\right| \\
& \leqq \\
& (n+1) !\left\{(n+2) x^{n+3}-(1-r) x^{n+2}\right\}(1-r)^{-n-8},
\end{aligned}
$$

the right side of the equation for ${ }_{0} D_{z}^{b} g(z)$ may be reduced to the bound given in the theorem by a procedure exactly analogous to that for $a<0$. Thus it remains necessary to show only that

From

$$
\left|{ }_{0} D_{z}^{b} g(z)\right|=\left|{ }_{0} D_{z}^{a} f(z)-\sum_{j=1}^{n}{ }_{0} D_{z}^{a-j} f^{(j)}(0)\right| .
$$

$$
{ }_{0} D_{z}^{b} f^{(n)}(z)=\frac{\Gamma(b+1)}{2 \pi i} \int_{P} f^{(n)}(w)(w-z)^{-b-1} d w
$$

we can obtain, using the proper determination of $(w-z)^{-b-1}$ and in- 
tegration by parts with $f^{(n)}(w) d w$ as the differential,

$$
{ }_{0} D_{z}^{b} f^{(n)}(z)=-\frac{1}{\Gamma(-b)} f^{(n-1)}(0) r^{-b-1} e^{-(b+1) i t}+{ }_{0} D_{z}^{b+1} f^{(n-1)}(z) .
$$

The process may be repeated to get

$$
{ }_{0} D_{x}^{b} f^{(n)}(z)={ }_{0} D_{x}^{a} f(z)-\sum_{j=0}^{n-1} \frac{1}{\Gamma(1-a+j)} f^{(j)}(0) r^{j-a} e^{i(j-a) t} .
$$

But

$$
{ }_{0} D_{z}^{a-j} f^{(j)}(0)=\frac{1}{\Gamma(1-a+j)} f^{(j)}(0) r^{i-a} e^{i(j-a) t}
$$

by direct evaluation, so

and

$$
{ }_{0} D_{z}^{b} f^{(n)}(z)={ }_{0} D_{z}^{a} f(z)-\sum_{j=0}^{n-1}{ }_{0} D_{z}^{a-j} f^{(j)}(0)
$$

since $f(0)=0$.

$$
\begin{aligned}
{ }_{0} D_{z}^{b} g(z)={ }_{0} D_{z}^{b} f^{(n)}(z)-{ }_{0} D_{z}^{b} f^{(n)}(0) & ={ }_{0} D_{z}^{a} f(z)-\sum_{j=0}^{n}{ }_{0} D_{z}^{a-j} f^{(j)}(0) \\
& ={ }_{0} D_{z}^{a} f(z)-\sum_{j=1}^{n}{ }_{0} D_{z}^{a-j} f^{(j)}(0),
\end{aligned}
$$

Since every relation in the entire proof, except those limiting the range of $a$ and $b$, becomes an equality for real positive $z$ when $f(z)$ $=z(1-z)^{-2}$, this completes the proof.

The result reduces to that of Marty for non-negative integral values of $a$.

\section{BIBLIOGRAPHY}

1. N. H. Abel, Oeuvres, vol. 1, Christiania, 1881, pp. 11-27.

2. L. Bieberbach, Lehrbuch der Funktionentheorie, vol. 2, Leipzig, 1931, pp. 76-80.

3. H. T. Davis, The theory of linear operators, Bloomington, 1936, pp. 64-76.

4. H. Laurent, Sur le calcul des dérivées d indices quelconques, Nouvelles Annales de Mathématiques vol. 3 (1884) p. 240.

5. J. E. Littlewood, On inequalities in the theory of functions, Proc. London Math. Soc. vol. 23 (1924) p. 481.

6. F. Marty, Sur les dérivées d'une fonction univalente, C. R. Acad. Sci. Paris vol. 194 (1932) p. 1308. 53-55.

7. P. Montel, Lę̧ons sur les fonctions univalentes ou multivalentes, Paris, 1933, pp.

8. K. Pearson, Tables of the incomplete beta function, Cambridge, 1934, p. vi.

9. B. Riemann, Werke, Leipzig, 1876, p. 353.

The University of Rochester 